\title{
A No-Nonsense Control Engineering Approach to Anaesthesia Control during Induction Phase
}

\author{
Robin DE KEYSER, Clara M. IONESCU
} Ghent University, EeSA-department of Electrical energy, Systems and Automation,
Technologiepark 913, B9052 Gent, Belgium (Robain.DeKeyser@UGent.be)

\begin{abstract}
This paper introduces a strategy for patient-individualized control of induction phase during general anaesthesia. A down-to-earth, pragmatic (and possibly provocative) approach has been used. The core of the paper is the idea to avoid the direct use of the Bispectral Index signal (BIS) as a feedback signal, since it introduces artificial delays, it is noisy and in the initial stage of the induction it contains no useful information for control purposes because it hardly varies and it introduces nonlinearity. The effect site concentration $\left(C_{e}\right)$ of the drug in the patient is instead used as the controller's feedback signal. Of course the BIS measurement is still indirectly used in our proposed control strategy, in order to adapt the parameters of the Hill curve - which relates BIS to $C_{e}$ - resulting in a patient-individualized closed loop control of anesthesia. These ideas are validated on 12 virtually generated patients varying significantly in sensitivity to the drug effect, using a simple PID control. The control of the induction phase is split into two phases: the initial phase where no useful BIS feedback signal is available, hence target controlled infusion (TCI) with a fixed setpoint is temporarily applied; and the secondary phase when a useful BIS feedback signal becomes available and the TCI setpoint is adapted based on the estimated Hill-curve steepness. The approach is simple and excellent simulation results combined with theoretical insights indicate that the strategy looks promising for future clinical practice.
\end{abstract}

Keywords: estimation, adaptation, PID control, linear control, anaesthesia

\section{INTRODUCTION}

Optimal sedation using a combination of hypnotics and analgesics has become an integral part of critical care medicine. However, standard dosing guidelines in the operation theatre often result in an inappropriate under- or over-sedation due to huge inter-patient pharmacological variability. Both under- and over-sedation can lead to increased morbidity and mortality (Kress et al, 2002). Manual patient-individualization of dosing schemes is actually not accurately applied due to lack of care resources. In order to individualize patient's sedation trajectory, pathways to apply automation technology in order to better take into account and control pharmacological variability among critical ill patients can improve the efficiency of delivery of care and can reduce resource utilization.

Several SISO control algorithms have been developed to control drug administration during anaesthesia, the first attempt being made as early as 1951 (Kiersey et al, 1951). The earliest anesthesia controllers use three-term controllers such as PID (Ritchie et al, 1987), which are by far the most commonly used type of fixed-gain controllers. In these cases it is assumed that all patients have similar response to the drug input, while the more recent work in this area (Gentilini et al, 2001) employs more sophisticated modelling approaches including multiple linear models and detailed non-linear compartment models of the patient. Due to the fact that PID controllers cannot anticipate to the response of the patient and do not have any knowledge of the drug metabolism, stability problems are present. Therefore, strategies using adaptive (Haddad et al, 2003; Yelneedi et al, 2009; Dumont et al, 2009), predictive (Nunes et al, 2007; Ionescu et al, 2008; Nino et al, 2009) and bayesian-based (De Smet et al, 2008; Struys et al, 2004; Mortier et al, 1998) closed-loop control algorithms have been suggested. Most of these controllers use the Bispectral Index (BIS) signal as a feedback signal for control. BIS is an indirect measure of the EEG activity of the brain and provides insight into the degree of hypnosis induced in the patient. When compared against target controlled infusion, which is in fact manual control (i.e. the clinical nurse makes changes in the desired effect site drug concentration based on the BIS readings), the closed loop control using BIS signal performed better in terms of drug efficiency (Struys et al, 2004).

However, the direct use of BIS as feedback signal poses some drawbacks from control engineering point-of-view: i) it is an indirect measure which introduces artificial and often varying time-delays into the system, originating from the signal processing algorithms used to extract the BIS values from the raw EEG signal (Ionescu et al, 2011); ii) it is a noisy signal; iii) it introduces a nonlinearity in the system and iv) during the initial phase of the induction it does not vary significantly, due to the sigmoid shape of the Hill curve.

This paper presents an alternative approach to computer control during the induction phase of general anaesthesia, such that the BIS signal is not directly used as a feedback signal. The solution proposed in this paper is to use the effect site concentration $\left(C_{e}\right)$ of the drug in the patient as a feedback 
signal and to estimate the steepness of the Hill curve (relating the BIS and $C_{e}$ ) during the induction phase, resulting in a patient-individualized closed loop control of anaesthesia. These ideas are validated on 12 virtually generated patients varying significantly in sensitivity to the drug effect (i.e. the parameters of the Hill curve) with a simple PID control whose information is based on variations in the effect site concentration.

The paper is organized as follows: the second section presents the structure of the patient compartmental model and the Hill curve parameters. The third section presents the proposed control strategy for the induction phase, along with the estimation algorithm of the required Hill curve characteristic parameters in order to obtain a patientindividualised control. The fourth section presents the results of the simulation analysis on the 12 patients and discusses the extreme patient cases: very sensitive or very resistant to drug effect. A conclusion section summarises the main outcome of this paper and motivates the applicability of this strategy into clinical practice.

\section{MODEL OF THE PATIENT FOR PROPOFOL INFUSION AND BIS EFFECT}

Propofol is a hypnotic agent, whose pharmacologic properties have been well described and studied in different kind of patients (Struys et al, 2004; Mortier et al, 1998). Propofol is used as a sedative agent in ICU, as well as the agent of choice for most short surgical procedures requiring transient unconsciousness. Propofol rate is the input of the model and the output is the Bispectral Index (BIS), a signal derived from the electroencephalogram (EEG). BIS values lie in the range of 0-100; whereas 90-100 range represents fully awake patients; 60-70 range and 40-60 range indicate light and moderate hypnotic state, respectively. For patient safety and fast recovery time, BIS should not decrease below 30 . The patient model is a compartmental model, known as the pharmacokinetics - pharmacodynamics (PK-PD) model. Compartmental models are used to represent the distribution of drugs in the body based on mass balance concepts. The transport rate that leaves the compartment is assumed to be proportional to the drug concentration. The PK-PD model most commonly used for Propofol is the $4^{\text {th }}$ order compartmental model described by Schnider (Schnider et al, 1998, 1999), with the structure depicted in Figure 1.

The PK-PD model is represented by the following equations:

$$
\begin{aligned}
\dot{x}_{1}(t) & =-\left[k_{10}+k_{12}+k_{13}\right] \cdot x_{1}(t)+k_{21} \cdot x_{2}(t) \\
& +k_{31} \cdot x_{3}(t)+u(t) / V_{1} \\
\dot{x}_{2}(t) & =k_{12} \cdot x_{1}(t)-k_{21} \cdot x_{2}(t) \\
\dot{x}_{3}(t) & =k_{13} \cdot x_{1}(t)-k_{31} \cdot x_{3}(t) \\
\dot{x}_{e}(t) & =-k_{e 0} \cdot x_{e}(t)+k_{1 e} \cdot x_{1}(t)
\end{aligned}
$$

where $x_{1}[\mathrm{mg} / \mathrm{ml}]$ denotes the drug concentration in the central compartment. The peripheral compartments 2 and 3 model the drug exchange of the blood with well and poorly diffused body tissues. The concentrations of drug in fast and slow equilibrating peripheral compartments are denoted by $x_{2}$ and $x_{3}$, respectively. The parameters $k_{j i}$ for $i \neq j$, denote the drug transfer frequency from the $j^{\text {th }}$ to the $i^{\text {th }}$ compartment and $u(t)[\mathrm{mg} / \mathrm{s}]$ is the infusion rate of the anesthetic drug into the central compartment. It is common practice to define an additional (virtual) effect compartment in order to represent the lag between plasma drug concentration and drug response. The concentration of drug in this compartment is represented by $x_{e}$. The effect compartment receives drug from the central compartment by a first-order process and it is regarded as a volumeless additional compartment. Therefore, the drug transfer frequency from the central compartment to the effect-site compartment is equal to the frequency of drug removal from the effect-site compartment: $k_{e 0}=k_{1 e}=0.456$ [min $\left.{ }^{-1}\right]$. The parameters $k_{i j}$ of the PK models depend on age, weight, height and gender and can be calculated for Propofol: $V_{1}=4.27\left[l \mid ; V_{2}=18.9-0.391 \cdot(\right.$ age -53$)|l| ; V_{3}=2.38[l]$ $C l_{1}=1.89+0.0456($ weight -77$)-0.0681(\mathrm{lbm}-59)$

$$
+0.0264(\text { height }-177)[l / \mathrm{min}]
$$

$\mathrm{Cl}_{2}=1.29-0.024$ (age -53$)\left[\mathrm{l} / \mathrm{min}, \mathrm{Cl}_{3}=0.836[\mathrm{l} / \mathrm{min}]\right.$

$k_{10}=\frac{C l_{1}}{V_{1}}\left[\min ^{-1}\right], k_{12}=\frac{C l_{2}}{V_{1}}\left[\min ^{-1}\right], k_{13}=\frac{C l_{3}}{V_{1}}\left[\min ^{-1}\right]$,

$k_{21}=\frac{C l_{2}}{V_{2}}\left[\min ^{-1}\right], k_{31}=\frac{C l_{3}}{V_{3}}\left[\min ^{-1}\right]$

where $C l_{1}$ is the rate at which the drug is cleared from the body, and $\mathrm{Cl}_{2}$ and $\mathrm{Cl}_{3}$ are the rates at which the drug is removed from the central compartment to the other two compartments by distribution. The lean body mass (lbm) for women and men are: $\mathrm{lbm}=1.1 \cdot$ weight $-128 \cdot \frac{\text { weight }^{2}}{\text { height }^{2}}$ and $\mathrm{lbm}=1.07 \cdot$ weight $-148 \cdot \frac{\text { weight }^{2}}{\text { height }^{2}}$ respectively.

The relation between the effect site concentration and the BIS is given by a nonlinear sigmoid Hill curve:

$$
B I S(t)=E_{0}-E_{\max } \cdot \frac{C_{e}^{\gamma}(t)}{C_{e}^{\gamma}(t)+C_{50}^{\gamma}}
$$

where $E_{0}$ is the BIS value when the patient is awake; $E_{\max }$ is the maximum effect that can be achieved by the infusion of Propofol; $C_{50}$ is the Propofol concentration at half of the maximum effect and $\gamma$ is a parameter which together with the $C_{50}$ determines the patient sensitivity to the drug. $E_{0}$ and $E_{\max }$ are usually considered equal to 100 . A (varying) dead-time added to (4) takes into account the time-delay introduced by the BIS-measurement as explained earlier.

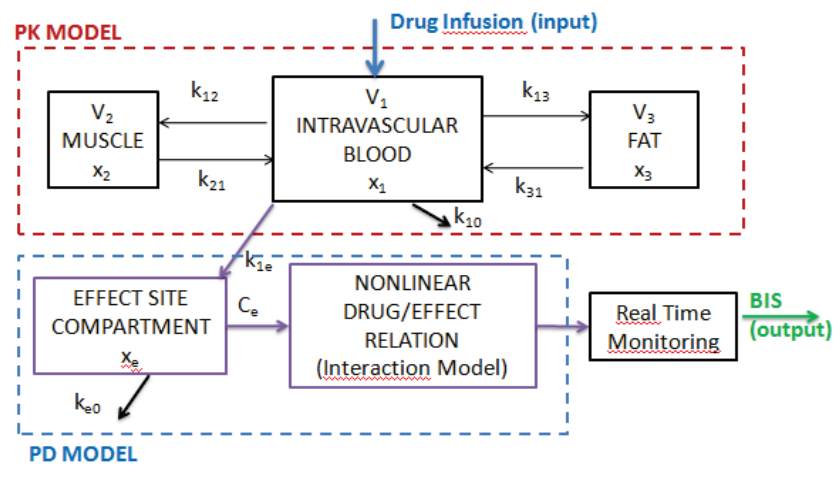

Figure 1: Compartmental scheme of the PK-PD model. 


\section{PROPOSED CONTROL STRATEGY DURING INDUCTION PHASE OF GENERAL ANAESTHESIA}

The strategy proposed here is summarized in Figure 2 below. The control of the induction phase is split into two phases: the initial phase where no useful BIS feedback signal is available, hence target controlled infusion (TCI) with a fixed setpoint is temporarily applied; and the secondary phase when a useful BIS feedback signal becomes available and the TCI setpoint is adapted based on the estimated Hill-curve steepness.

\subsection{First Part of Induction: Target Controlled Infusion}

One may observe in figure 2 the typical sigmoid shape of the Hill curve (4) relating the BIS values to the effect site concentration $C_{e}$, which starts with a flat plateau (i.e. also called dead-zone).

Claim: during this time, no closed loop feedback control can be applied since there is no useful feedback signal available - the Propofol drug is infused into the patient while BIS values do not vary significantly; moreover in practice the little variation is hidden in noise.

The only solution is to apply target controlled infusion (TCI) until BIS values show a certain variation $\triangle \mathrm{BIS}$. TCI is currently applied in clinical practice using a target effect concentration (setpoint $C_{e}^{*}$ ) which ideally should be close to $C_{50}$ if the desired BIS=50. Unfortunately $C_{50}$ is unknown and patient-dependent. For safety reasons, it is logical to use the minimum expected $C_{50}$ ( $=5$ in our experiments, ref. Table 1$)$.

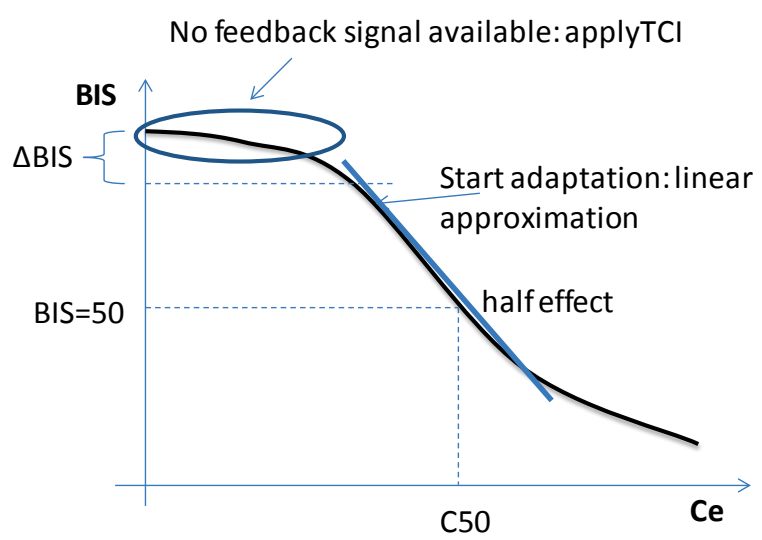

Figure 2: Schematic representation of the proposed control strategy during induction phase

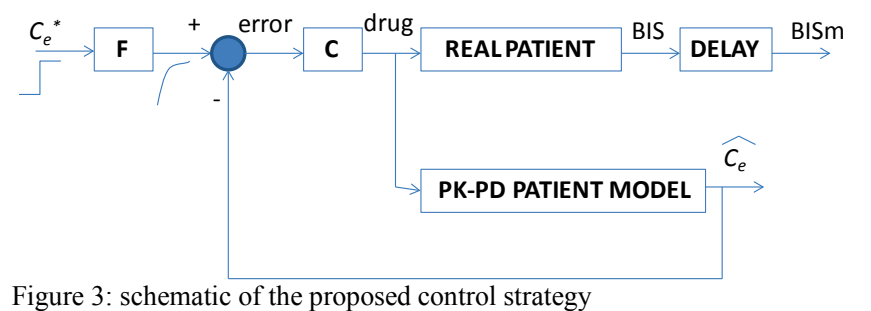

\subsection{Second Part of Induction: Closed loop PID control}

At the moment that a variation of $\Delta \mathrm{BIS}$ is achieved, then one can switch from TCI to closed loop control. A first intuitive choice is to use the measured BIS value as feedback information. However, this choice implies some disadvantages from control engineering point of view: i) it introduces nonlinearity in the loop (through the Hill curve); ii) it introduces variable time-delays originating from the signal processing of the raw EEG signal - this challenges the stability of the closed loop; and iii) it is a noisy signal which will increase the control effort through the derivative action this can be avoided by filtering techniques, with the undesired side-effect of introducing phase lags in the loop.

It is therefore meaningful to continue to use the estimated $\widehat{C_{e}}$ from the $4^{\text {th }}$ order PK-PD model given in (1)(2). The advantages of using it as a feedback signal are i) it is not noisy; ii) it does not introduce artificial time delays; iii) it is linear and iv) the transfer function of the 'controlled process' is known (the PK-PD patient model) which makes it rather straightforward to tune the PID controller. Notice that in our experimental results of section 4, only 1 nominal PK-PD model and 1 corresponding PID controller was used for the control of all 12 patients in Table 1 . The mean values of the PK-PD models in Table 1 were used as nominal model, so no a-priori individualized patient information is needed. The general control scheme corresponding to this reasoning is depicted in Figure 3 below. In this scheme, the PID controller is denoted by the block $\mathbf{C}$ and the block $\mathbf{F}$ is a low pass filter which avoids the effects of the derivative kick when the reference for $C_{e}{ }^{*}$ is changing (i.e. smooth transition).

\subsection{Estimation of some Hill curve characteristics for patient-individualized anaesthesia control}

Until the switching moment, the target effect concentration $C_{e}^{*}$ is fixed at a safe value ( 5 in our experiments). After the switch, this value is adapted based on the following ideas. Since the most important challenge for control is the interpatient variability, it makes sense to estimate some useful parameters of the actual patient's Hill curve. Observing Figure 2 again it is clear that around the target value $\mathrm{BIS}=50$, the Hill curve can be approximated by a linear model (i.e. a straight line). The available measured BIS values (denoted by $B I S m$ in Figure 3 ) and the calculated $\widehat{C_{e}}$ from the $4^{\text {th }}$ order PK-PD model are related by the linear approximation and a time delay:

$$
\operatorname{BISm}(t)=K * \widehat{C_{e}}(t-\tau)+d
$$

where the $K, d$ and $\tau$ are estimated at every sampling instant. An easy procedure to do so consists of: 1) preselect a set of plausible time-delay values $\tau$; 2) estimate for every timedelay value the corresponding parameters $K$ and $d$ with a least-squares algorithm; 3) compare the variance of the estimation errors to select the best set $[K, d, \tau]$.

The target effect concentration $C_{e}^{*}$ is then adapted according to:

$$
C_{e}^{*}=\left(B I S^{*}-\hat{d}\right) / \widehat{K}
$$

with $B I S^{*}$ the target BIS value. In fact, for $B I S^{*}=50$, this is nothing else than the estimation of the patient's $C_{50}$.

Notice that the adaptation of the $K$ and $d$ parameters allows also for some errors in the used PK-PD model. Indeed any error in its static gain will be corrected intrinsically by the estimation of the Hill curve steepness. Errors in the dynamical parameters however have to be tackled by the robustness of the PID controller. 


\subsection{Analysis of the Hill curve parameters}

Consider again the form of the sigmoid Hill curve from (4). Introducing $X=\frac{C_{e}}{C_{50}}$, we have that $X^{\gamma}=\frac{E_{0}-E}{E_{\max }-E_{0}+E}$, with $E$ the effect of the drug (i.e. BIS). The derivative, i.e. the slope of the nonlinear curve, is given by:

$$
\frac{d E}{d X}=-E_{\max }\left[\frac{\gamma X^{\gamma-1}}{1+X^{\gamma}}-\frac{X^{\gamma} \gamma X^{\gamma-1}}{\left(1+X^{\gamma}\right)^{2}}\right]=-E_{\max } \gamma \frac{X^{\gamma-1}}{\left(1+X^{\gamma}\right)^{2}}(7)
$$

Using $\frac{X^{\gamma}}{1+X^{\gamma}}=\frac{E_{0}-E}{E_{\max }}$, it follows that the derivative with respect to $C_{e}$ can be written as:

$$
\frac{d E}{d C_{e}}=\frac{d E}{d X} \frac{d X}{d C_{e}}=\frac{-E_{\max } \gamma}{C_{50}} \frac{1}{X^{\gamma+1}}\left[\frac{X^{\gamma}}{1+X^{\gamma}}\right]^{2}
$$

and using $X=\frac{C_{e}}{C_{50}}$ we obtain:

$$
\frac{d E}{d C_{e}}=\frac{-\gamma}{C_{e}} \frac{\left(E_{\max }-E_{0}+E\right)\left(E_{0}-E\right)}{E_{\max }}
$$

For the case $E_{\max }=E_{0}=100$ it follows that

$$
\frac{d E}{d C_{e}}=\frac{-\gamma}{C_{e}} \frac{E(100-E)}{100}
$$

and for the case $\mathrm{BIS}=\mathrm{E}=50$ we have that the slope is proportional to $-\frac{\gamma}{C_{50}}$.

The slope at $\mathrm{BIS}=100$ is zero and it is easy to show that the slope of the flat plateau in Fig. 2 near $B I S=100$ is proportional to $-\frac{\gamma}{C_{50}^{\gamma}}$ (e.g. take a small value for $C_{e}$ ). These slopes are depicted in Figure 4 as a function of $\gamma$ and $C_{50}$.
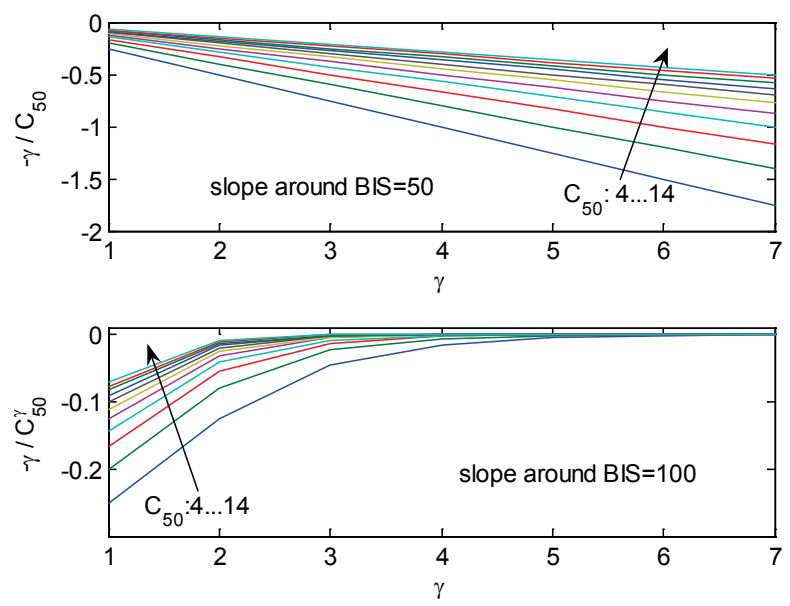

Figure 4: Slopes of the Hill curve.

\subsection{PID Control Design}

The PID tuning algorithm is based on imposing a userspecified robustness to the control loop (De Keyser et al, 2012). The robustness specification can be translated as a circle with a specified radius $r$ around the critical point -1 in the Nyquist plane ( $r=$ modulus margin; $0<r<1 ; r=0.5$ in the experiments of Section 4). In order to obtain a fluent curve for the frequency response of the controller and the process $\left(C^{*} \mathrm{P}\right)$, going smoothly around the specified circle, we impose that at the critical frequency $\omega_{c}$ of the process (i.e. where the phase lag is $-180^{\circ}$ ), the $\mathrm{C}^{*} \mathrm{P}$ curve will be tangent to the robustness circle in a point $\mathrm{A}$. This results in:

$$
\begin{gathered}
M_{A} * e^{j \varphi_{A}}=M_{P * C}\left(j \omega_{C}\right) * e^{j \varphi_{P * C}\left(j \omega_{C}\right)} \\
\left.\frac{d M}{d \varphi}\right|_{A}=\left.\frac{d M_{P * C}}{d \varphi_{P * C}}\right|_{\omega=\omega_{C}}
\end{gathered}
$$

That is: the modulus and the phase at point $\mathrm{A}$ should be equal to the modulus and the phase of the process plus controller at $\omega_{c}$. Also, the value of the derivative to the circle in A should be equal to the derivative of the $\mathrm{C} * \mathrm{P}$ frequency response at $\omega_{c}$. The vector from the critical point -1 to the tangent point A has length $r$ and an angle $\alpha$ with the negative real axis. In (De Keyser et al, 2012) an algorithm has been described to find the angle $\alpha^{*}$ for which the error $\left|\frac{d M}{d \varphi}\right|_{A}-\left.\frac{d M_{P * C}}{d \varphi_{P * C}}\right|_{\omega=\omega_{C}} \mid$ is minimum. The PID parameters are then:

$$
K_{p}=\frac{1-r \cos \alpha^{*}}{M_{p}\left(j \omega_{c}\right)}, \quad T_{d}=\frac{M_{A}\left(\alpha^{*}\right)+r \sin \alpha^{*}}{2 \omega_{c}\left(1-r \cos \alpha^{*}\right)}, \quad T_{i}=4 T_{d}
$$

The necessary process information such as the critical frequency $\omega_{c}$, the value of the process modulus $M_{p}\left(j \omega_{c}\right)$ and phase $\varphi_{p}\left(j \omega_{c}\right)$, the value of the derivatives at $\omega_{c}$, can be obtained from the process transfer function (which is in this case a nominal version of the patient model (1)(2)).

Notice that other compartmental mixing models than (1)(2) have been described in literature. Our proposed patientindividualized control strategy described in sections 3.1, 3.2, 3.3 is equally valid for such alternative models.

\section{SIMULATION RESULTS}

In all experiments, the sampling period has been 1 second. For illustrating the proposed strategy, the virtual patients from (Ionescu et al, 2008) have been used as presented in Table 1. A nominal patient model is defined by using the mean value of all these parameters; only the mean values for 'Age, Length, Weight, Gender' are used to design the PID (i.e. the values of $\gamma$ and $C_{50}$ are never used in our control strategy). For safety, a low value $C_{e}{ }^{*}=5 \mathrm{mg} / \mathrm{mL}$ is used during the initial TCI strategy. At the switching moment (i.e. when BIS=90), the adaptation strategy adapts the setpoint $C_{e}{ }^{*}$ in

\begin{tabular}{|c|c|c|c|c|c|c|}
\hline : & $\underset{\&}{\infty}$ & 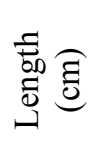 & 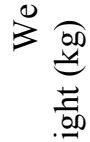 & $\frac{\bar{\theta}}{\dot{0}}$ & $\mathrm{C}_{50}$ & $\gamma$ \\
\hline 1 & 40 & 163 & 54 & $\mathrm{M}$ & 6.33 & 2.24 \\
\hline 2 & 36 & 163 & 50 & $\mathrm{M}$ & 6.76 & 4.29 \\
\hline 3 & 28 & 164 & 52 & $\mathrm{M}$ & 8.44 & 4.10 \\
\hline 4 & 50 & 163 & 83 & $\mathrm{M}$ & 6.44 & 2.18 \\
\hline 5 & 28 & 164 & 60 & $\mathrm{~F}$ & 4.93 & 2.46 \\
\hline 6 & 43 & 163 & 59 & $\mathrm{M}$ & 12.0 & 2.42 \\
\hline 7 & 37 & 187 & 75 & $\mathrm{~F}$ & 8.02 & 2.10 \\
\hline 8 & 38 & 174 & 80 & $\mathrm{M}$ & 6.56 & 4.12 \\
\hline 9 & 41 & 170 & 70 & $\mathrm{M}$ & 6.15 & 6.89 \\
\hline 10 & 37 & 167 & 58 & $\mathrm{M}$ & 13.7 & 1.65 \\
\hline 11 & 42 & 179 & 78 & $\mathrm{~F}$ & 4.82 & 1.85 \\
\hline 12 & 34 & 172 & 58 & $\mathrm{M}$ & 4.95 & 1.84 \\
\hline Mean & 38 & 169 & 65 & M & 7.42 & 3.00 \\
\hline
\end{tabular}
order to converge to the true $C_{50}$ value for each patient.

The Hill curves of all patients are given in Figure 5. The $C_{50}$ values as well as the values of $-\frac{\gamma}{C_{50}}$ and $-\frac{\gamma}{C_{50}^{\gamma}}$ are given in Figure 6; these last values are proportional to the slopes of the Hill curve around BIS=50 and BIS $=100$.

Table 1. Patient Parameters 


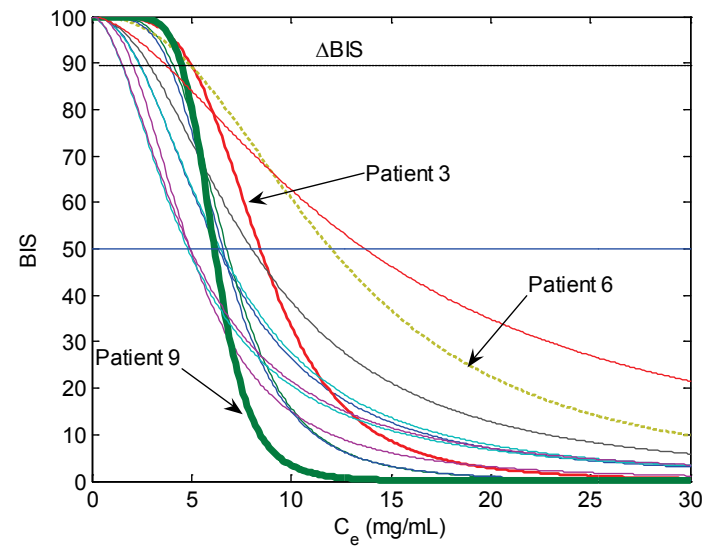

Figure 5: Hill curves for all patients from Table 1. See text for comments.
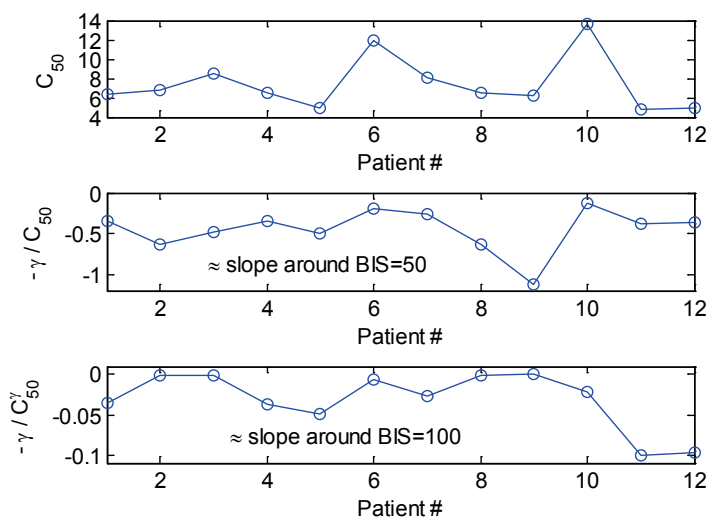

Figure 6: $\mathrm{C}_{50}$ values and Hill slopes around $\mathrm{BIS}=50$ and $\mathrm{BIS}=100$.

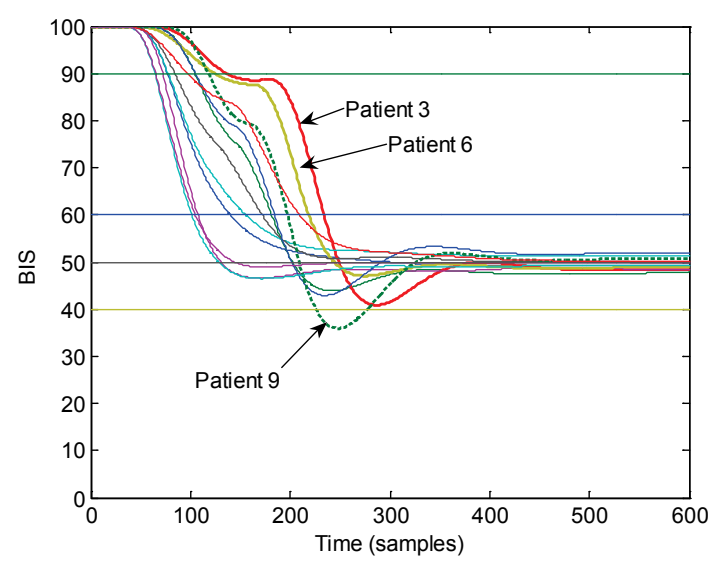

Figure 7: Closed loop response for all patients from Table 1. In all time signals, 1 sample corresponds to 1 second.

\subsection{Detailed results for patient 7}

Let us now look at the convergence of the $K, d$ and the error during the estimation interval, depicted in Figure 8. The $K$ and $d$ parameters which give the lowest error are selected and used in the closed loop control, as depicted in Figure 9. Based on these, the setpoint $C_{e}^{*}$ is adapted and Propofol values updated, as illustrated in Figure 10. It can be observed that the BIS values are converging to the desired value of 50 without undershoot and in very short period of time (about 3 minutes). The dashed vertical line in Figure 10 indicates the switching moment. The real time-delay used in the experiments was 30s. Notice that the $C_{e}^{*}$ setpoint value depicted in Figure 10 is the filtered signal from Figure 9, thus avoiding unwanted effects from possible estimation jumps. In Figure 10 can also be observed the convergence from the nominal value of $C_{e}{ }^{*}=5$ to the true $C_{50}=8$ (Patient 7, Table 1). It is also important to check the robustness against a significant amount of noise present in the BIS signal. The results for Patient 7 in the presence of noise $(+-10 \%$ BIS) are given in Figures 11-12. It can be observed that the adaptation algorithm converges very well to the true values. Because the noisy BIS signal is not directly used in the feedback loop rather indirectly via the setpoint $C_{e}{ }^{*}$ - the Propofol and $C_{\mathrm{e}}$ signals are almost noise-free!
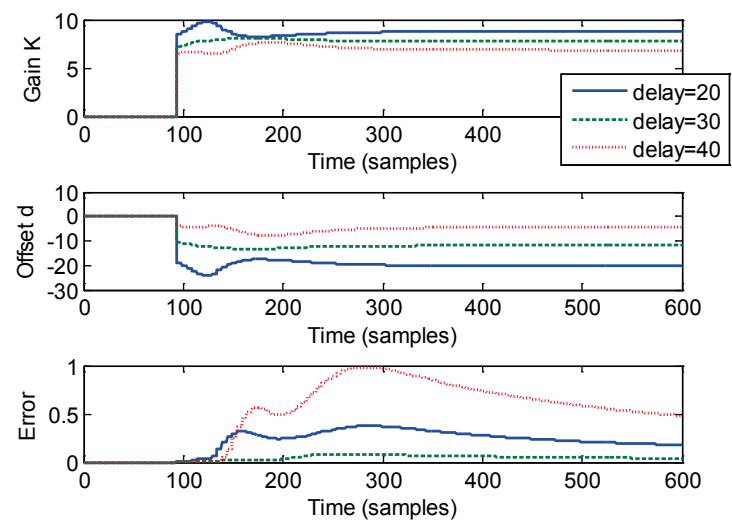

Figure 8: Illustrative example of the estimation of the $K$ and $d$ parameters for different values of the delay. The error is calculated and the minimum error will indicate which parameters to be used in the control scheme.
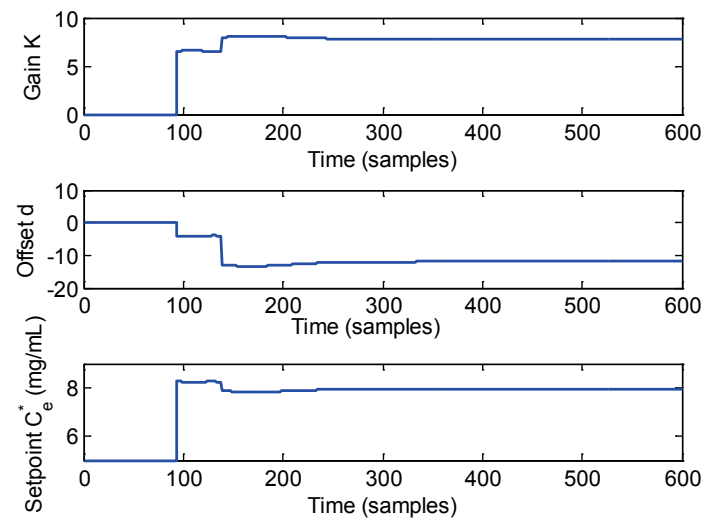

Figure 9: End-result of the adaptation procedure for the $K$ and $d$ parameters and the consequent adaptation of the setpoint.

\subsection{Further insights}

Some of the patients $(2,3,8,9)$ suffer from some undershoot in the BIS (Figure 7). Although not being a real problem from practical point-of-view, a theoretical explanation would nevertheless be welcome. A small slope around $\mathrm{BIS}=100$ (i.e. a long flat plateau in Figure 2) results in a $C_{\mathrm{e}}$-value which is already rather big at the moment of switching. If this phenomenon is accompanied with a $C_{50}$-value which is not much bigger, it may result in undershoot because there is not much time for good estimation (as is well-known, good parameter estimation requires a sufficient amount of data). 


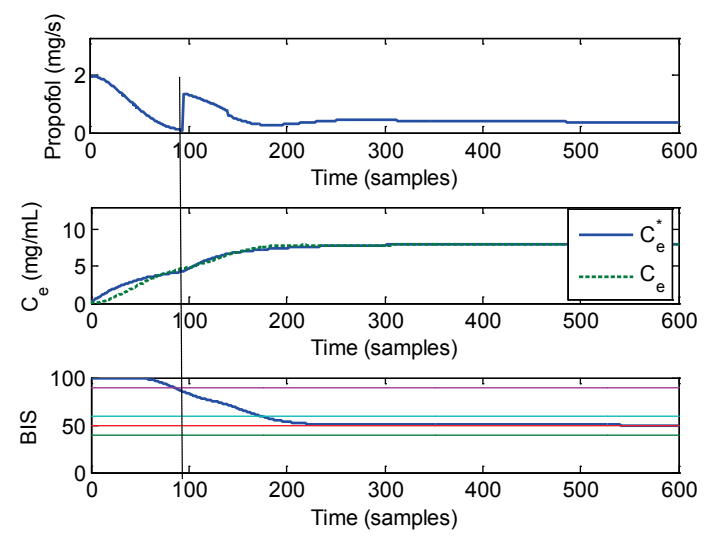

Figure 10: The Propofol infusion rate, the filtered setpoint $C_{e}{ }^{*}$, the calculated $C_{e}$ from the nominal PK-PD model used in the control and the BIS value.
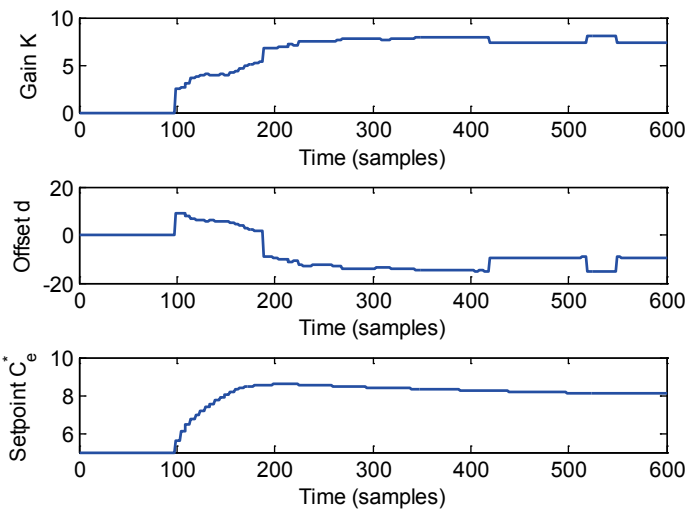

Figure 11: Estimation in the presence of noise: end-result of the adaptation procedure for the $K$ and $d$ parameters and the consequent adaptation of the setpoint.

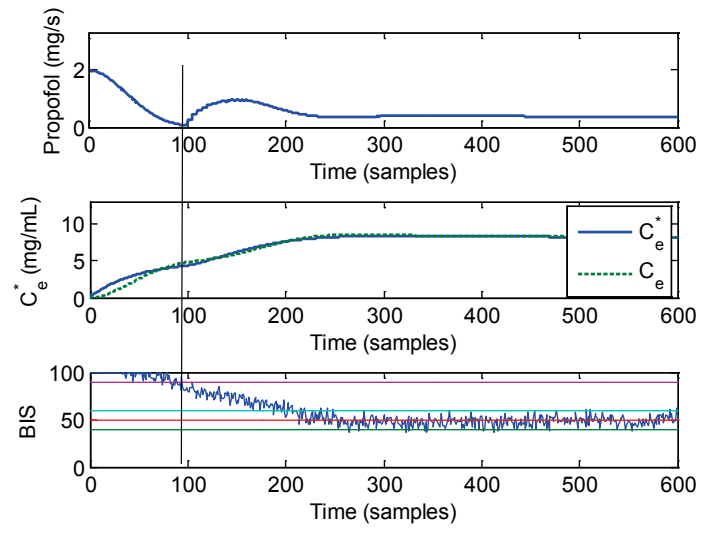

Figure 12: Control in the presence of noise: the Propofol infusion rate, the filtered setpoint $C_{e}{ }^{*}$, the calculated $C_{e}$ from the nominal PK-PD model used in the control and the BIS value.

A small slope around $\mathrm{BIS}=100$ is produced by a big value of $\gamma$ (Figure 4, lower part). Such $\gamma$ then also results in a big slope around $\mathrm{BIS}=50$, unless $C_{50}$ is large (Fig. 4, upper part), and this then leads to a fast transfer to low BIS values with little time for estimation. This can also be observed in Figure 5, e.g. patient 9. Figure 6 shows that patients $2,3,6,8,9$ are indeed characterized by such small slope around BIS $=100$. Patient 6 however has a big $C_{50}$, which explains why the BIS does not have undershoot. This diagnosis of the problem also produces the remedy: if the $C_{\mathrm{e}}$-value at the moment of switching is already high, then be cautious and change only gradually the target effect concentration $C_{e}^{*}$ that is produced by the estimator. This can be easily done by using a $1^{\text {st }}$-order $C_{e}^{*}$-filter with time constant depending on the value of $C_{\mathrm{e}}$ at the switching moment. On the other hand, this problem is probably more of academic than of practical interest, because the values of $\gamma$ which have been published in literature are not as high as the ones used in our experiments (Dumont et al, 2009; Yelneedi et al, 2009).

\section{REFERENCES}

De Keyser R., Joita O.L., Ionescu C.M., (2012) "The Next Generation of Relay-based PID Autotuners (PART 2): A Simple Relay-based PID Autotuner with Specified Modulus Margin”, IFAC Conf. on Advances in PID Control (PID12), Brescia,

De Smet T., Struys MM., Neckbroek MM., Van den Hauwe K., Bonte S., Mortier E.P., (2008) "The accuracy and clinical feasibility of a new bayesian-based closed-loop control system for propofol administration using the bispectral index as a controlled variable", Anesth Analg, 107:1200-1210

Dumont G.A., Martinez A., Ansermino M. (2009) "Robust control of depth of anesthesia", in special issue "Trust me I am a doctor" of the Int Journal of Adaptive Control and Signal Processing, 23:435-454

Kiersey D.K, Bickford R.G, Faulconer Jr. A., (1951) "Electroencephalographic patterns produced by thiopental sodium during surgical operations: description and classification", British Journal of Anaesthesia, 23: 141-152

Kress JP, Pohlman AS, Hall JB, (2002) "Sedation and analgesia in the intensive care unit", Am J Respir Crit Care Med, 166: 1024-8

Gentilini A., Frei C.W., Glattfedler A.H., Morari M., Sieber T.J., Wymann R., Schnider T.W., Zbinden A.M., (2001) "Multitasked Closed-Loop Control in Anesthesia", IEEE Engineering in Medicine and Biology, 20: 39-53

Haddad W.M, Hayakawa T., Bailey J.M., (2003) "Nonlinear adaptive control for intensive care unit sedation and operating room hypnosis", American Control Conference, 1808-1813

Ionescu C.M., De Keyser R., Torrico B.C., De Smet T., Struys M., NormeyRico J.E., (2008) "Robust Predictive Control Strategy Applied for Propofol Dosing using BIS as a Controlled Variable during Anesthesia", IEEE Transactions on Biomedical Engineering, 55: 2161-2170

Ionescu C.M., Hodrea R., De Keyser R., (2011) "Variable Time Delay Estimation for Anesthesia Control during Intensive Care", IEEE Transactions on Biomedical Engineering Journal, 58(2): 363-369.

Mortier E., Struys M., De Smet T., Versichelen L., Rolly G., (1998) “Closedloop controlled administration of propofol using bispectral analysis", Anaesthesia, 53:749-754

Nino J., Syafiie S., De Keyser R., Ionescu C.M., Struys M..(2009) EPSAC Controlled anesthesia with online gain adaptation, in special issue "Trust me I am a doctor" of the Int Journal of Adaptive Control and Signal Processing, 23:455-471

Nunes C.S., Mendoca T., Lemos J.M., Amorim P., (2007) "Predictive Adaptive Control of the Bispectral Index of the EEG (BIS): Exploring Electromyography as an Accesible Disturbance", Proceedings of the $15^{\text {th }}$ Mediterranean Conference on Control and Automation, Athena, Greece

Ritchie R.G., Ernst E.A., Pate B.L., Pearson J.D., Sheppard L.C., (1987) "Closed-loop control of an anesthesia delivery system: Development and animal testing", IEEE Transactions on Biomedical Engineering, 34(6): 437-443

Schnider T.W., Minto C.F., Gambus P.L. , Andresen C, Goodale DB , Youngs EJ, (1998) "The influence of method of administration and covariates on the pharmacokinetics of Propofol in adult volunteers", in Anesthesiology, 88: 1170-1182

Schnider TW., Minto CF, Shafer SL, Gambús PL, Andresen C, Goodale DB, Youngs EJ, (1999) "The influence of age on Propofol pharmacodynamics", Anesthesiology, 90:1502-16

Struys MM., De Smet T., Greenwald S., Absalom AR., Binge S., Mortier E.P, (2004) "Performance evaluation of two published closed-loop control systems using bispectral index monitoring: a simulation study", Anesthesiology, 100: 640-647

Yelneedi S., Samavedham L., Rangaiah G.P. (2009) “Advanced control strategies for the regulation of hypnosis with Propofol", Ind. Eng. Chem. Res. 48:3880-3897 